\title{
Clinicopathological significance of BTF3 expression in colorectal cancer
}

Chao-Jie Wang, Hanna Frånbergh-Karlson, Da-Wei Wang, Gunnar Arbman, Hong Zhang and Xiao-Feng Sun

\section{Linköping University Post Print}

\section{Tweet}

N.B.: When citing this work, cite the original article.

The original publication is available at www.springerlink.com:

Chao-Jie Wang, Hanna Frånbergh-Karlson, Da-Wei Wang, Gunnar Arbman, Hong Zhang and Xiao-Feng Sun, Clinicopathological significance of BTF3 expression in colorectal cancer, 2013, Tumor Biology, (34), 4, 2141-2146.

http://dx.doi.org/10.1007/s13277-013-0745-8

Copyright: Karger / Springer Verlag (Germany) http://www.springerlink.com/?MUD=MP

Postprint available at: Linköping University Electronic Press http://urn.kb.se/resolve?urn=urn:nbn:se:liu:diva-96384 


\section{Clinicopathological significance of BTF3 expression in colorectal}

\section{cancer}

\section{Chao-Jie Wang· Hanna Frånbergh-Karlson· Da-Wei Wang· Gunnar Arbman· \\ Hong Zhang· Xiao-Feng Sun}

\section{J. Wang}

Department of Oncology, Henan Provincial People’s Hospital \& People’s Hospital of Zhengzhou University, Zhengzhou, China; Division of Oncology, Department of Clinical and Experimental Medicine, Faculty of Health Sciences, University of Linköping, Linköping, Sweden

H. Frånbergh-Karlson

Division of Oncology, Department of Clinical and Experimental Medicine, Faculty of Health Sciences, University of Linköping, Linköping, Sweden

D. W. Wang.

Department of Stomatology, the Third Hospital of Hebei Medical University,

Shijiazhuang, China

G. Arbman

Department of Surgery, Vrinnevi Hospital, University of Linköping, Norrköping, Sweden

H. Zhang

School of Medicine, Örebro University, Örebro, Sweden

X. F. Sun

Division of Oncology, Department of Clinical and Experimental Medicine 
Faculty of Health Sciences, County Council of Östergötland, University of Linköping, Linköping, Sweden

Running title: BTF3 in colorectal cancer

*Correspondence to: X. F. Sun, Prof., MD, PhD

Division of Oncology, Department of Clinical and Experimental Medicine, Faculty of Health Sciences, Country Council of Östergötland, University of Linköping, S-581 85 Linköping, Sweden

Tel: +46-(0)10-1032066, E-mail: xiao-feng.sun@liu.se 


\section{Abstract}

Basic transcription factor 3 (BTF3) is a general RNA polymerase II transcription factor, and also involved in apoptosis regulation. Increasing evidence shows that BTF3 is aberrantly expressed in several kinds of malignancies, but there is no study to analyze BTF3 expression in colorectal cancer (CRC) patients. Applying immunohistochemistry, we detected BTF3 in CRCs $(n=156)$, the corresponding distant $(n=42)$, adjacent normal mucosa $(n=96)$, lymph node metastases $(n=35)$, and analyzed its relationships with clinicopatholocial and biological variables. Our results showed that BTF3 staining significantly increased from distant or adjacent normal mucosa to primary CRCs $(p<0.0001)$ or metastases ( $p=0.002$ and $p<0.0001)$. BTF3 was higher in distal cancers than in proximal cancers ( $57 \%$ vs. $39 \%, p=0.041)$. It also showed stronger staining in primary CRCs stage I and II than that in stage III and IV (64\% vs. 35\%, $p=0.0004)$, or metastases ( $64 \%$ vs. $29 \%, p=0.004)$. Cancers with better differentiation had a higher expression than those with worse differentiation ( $56 \%$ vs. 37\%, $p=0.031$ ). There were positive correlations of BTF3

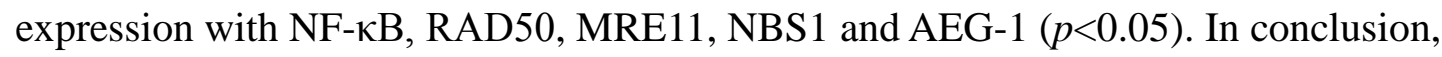
BTF3 overexpression may be an early event in CRC development, and could be useful biomarker for the early stage of CRCs. BTF3 has positive correlations with

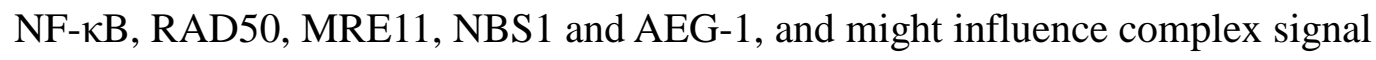
pathways in CRC. 
Wang CJ et al.

Key words Basic transcription factor $3 \cdot$ Biomarker $\cdot$ Colorectal

cancer $\cdot$ Immunohistochemistry 


\section{Introduction}

Basic transcription factor 3 (BTF3), a 27 KD protein, is a general RNA polymerase II transcription factor. It is evolutionary conserved and presents in a variety of mammalian cells [1, 2]. Its biological importance is shown by the fact that an insertional mutation in the BTF3 leads to an early postimplantation lethality in mice [3]. In addition to its function as a transcriptional regulator, BTF3 is also involved in cell apoptosis. Down-regulation of BTF3 is involved in inhibition of transcription and protein synthesis in the apoptotic K562 cells induced by harringtonine [4], and altered BTF3 expression is associated with apoptosis in BL60-2 Burkitt lymphoma cells [5].

More and more evidence shows the aberrant BTF3 expression in different tumors. In human brain astrocytomas, BTF3 is more highly expressed in glioblastoma multiforme, the highly infiltrating and aggressive high-grade, than that in the indolent low-grade form [6]. BTF3 is overexpressed in nasopharyngeal carcinoma [7] and pancreatic ductal adenocarcinoma (PDAC) [8]. More recently, BTF3 is also found overexpressed in transitional endoplasmic reticulum isolated from dissected liver tumor nodules of aflatoxin B1-treated rats [9].

BTF3 is overexpressed in some colon cancer cell lines [10], however, as both transcription factor and apoptosis related protein, the expression pattern is still unknown in colorectal cancer (CRC) patients. In the present study, applying immunohistochemistry, we examined BTF3 expression in 156 CRCs samples, and analyzed its relationships with clinicopathological variables and other molecular 
Wang CJ et al.

markers. 


\section{Materials and methods}

Patient samples

Formalin-fixed paraffin-embedded sections consecutively collected from 156 Swedish patients with primary CRC were used for immunohistochemical staining. The patients underwent surgical resection at Linköping Hospital, Linköping or Vrinnevi Hospital, Norrköping, between 1977 and 2001. Immunohistochemical staining also included corresponding distant normal mucosa specimens (taken from the margin of distant resection, which were histological free from pretumor and tumor, $n=42$ ), adjacent normal mucosa specimens (normal mucosa adjacent to primary tumor, $n=96$ ) and metastases in the regional lymph node $(n=35)$. This study was carried out with Medical Ethical Committee approval and informed consent in writing was obtained from each patient. The patients' gender (84 males and 72 females), age (range from 42 to 95, mean age 71 years), tumor location, stage, differentiation were obtained from surgical and pathological records from the hospitals. Tumors from ascending and transverse colon were graded as proximal while tumors from descending colon and rectum were considered as distal. Tumor differentiation was graded as better (including good and moderate tumors) or worse (including poor, mucinous and signet-ring cell tumors).The patients were followed until April 2005 and by that time, 55 patients had died due to the CRC.

Expression of the nuclear factor kappa B p65 phosphorylated at serine-536 
(NF-kB), RAD50, MRE11, NBS1 and astrocyte elevated gene-1 (AEG-1) examined by immunohistochemistry, were taken from previous studies carried out at our laboratory. There was no information available about tumor location in three patients, stage in four, growth pattern in eight, and differentiation in one.

Immunohistochemical assay

The immunohistochemical staining was performed on $5 \mu \mathrm{m}$ sections from paraffin embedded tissue. The sections were incubated in oven at $60{ }^{\circ} \mathrm{C}$ for $12 \mathrm{~h}$, then deparaffinized in xylene and hydrated in descending concentrations of ethanol. The sections were put in Tris-EDTA buffer (pH 9.0) and heated to $125{ }^{\circ} \mathrm{C}$ for 30 seconds, then cooled down to $90{ }^{\circ} \mathrm{C}$ for 10 seconds in high pressure cooker to retrieve the epitopes in the sections. This was followed by washing in phosphate-buffered saline (PBS, $\mathrm{pH}$ 7.4). Endogenous peroxidase activity was blocked with $3 \% \mathrm{H}_{2} \mathrm{O}_{2}$ in methanol followed by three washes in PBS. After blocking unspecific interactions with protein blocking solution, the primary polyclonal rabbit antibody against BTF3 (Santa cruz biotechnology, inc.) was added at 1:200 in antibody diluent (Dako Cytomation, Glostrup, Denmark). This was followed by incubation over night at $4{ }^{\circ} \mathrm{C}$. The second day the sections were incubated with HRP-conjugate labelled polymer anti-rabbit secondary antibody (Dako), followed by subjecting to 3, 3'-diaminobenzidine tetrahydrochloride (Dako), and finally the sections were counterstained with haematoxylin. For the negative control, IgG1 (Sigma-Aldrich, St. 
Louis, MO) was used instead of the primary antibody.

The sections were microscopically examined and scored independently by two investigators without any knowledge of the clinicopathological or biological data. The cytoplasmic staining intensity and nuclear staining percentage were determined for distant, adjacent normal mucosa, primary CRCs, and lymph node metastases. The cytoplasmic staining intensity was scored as negative ( $<5 \%$ positive cells), weak, moderate or strong. The nuclear staining percentage was scored as $<5 \%, 5-24 \%$, $25 \%-49 \%, 50-74 \%$, or $\geq 75 \%$. The cases with discrepant scoring were re-evaluated individually until both investigators agreed on the scoring. The remaining were re-examined by the two investigators together using a dual-headed microscope in order to reach a consensus score. To avoid artificial effects, areas with necrosis, poor morphology, or in the margins of the sections, were not considered.

\section{Statistical analysis}

The $\chi^{2}$ test or Fisher's exact test were used to determine differences of BTF3 expression among normal mucosa, primary CRCs and lymph node metastases, as well as relationships of BTF3 expression in primary CRCs with clinicopathological variables. Spearman correlation test was used to analyze the correlations of BTF3 with other molecular markers taken from previous studies performed at our laboratory. Cox’s proportional hazard model was used to test relationship between BTF3 expression and patient survival. The Kaplan- Meier method was used to calculate survival curves. Two-sided $p$-values of $<0.05$ were considered statistically 
Wang CJ et al.

significant. 


\section{Results}

BTF3 expression in normal mucosa, primary tumors and lymph node metastases

BTF3 staining was mainly present in the cytoplasm of normal epithelial and tumor cells (Fig. 1). Because of the clinicopathological similarities, we combined the negative, weak and moderate cytoplasmic staining intensity samples as a weak group, and the strong staining intensity as a strong group for further analyses. The frequency of strong BTF3 staining significantly increased from distant or adjacent normal mucosa to primary CRCs ( $2 \%$ vs. $51 \%, 0$ vs. $51 \%$, $p<0.0001$, Table 1 ) or metastases (2\% vs. $29 \%, p=0.002$; and 0 vs. $29 \%, p<0.0001$ ). Although BTF3 increased in metastases compared with distant or adjacent normal mucosa, it showed weaker staining when compared with primary CRCs ( $29 \%$ vs. $51 \%, p=0.018$, Table 1$)$. The intensity of BTF3 staining did not significantly differ between distant and adjacent normal mucosa ( $2 \%$ vs. $0, p=0.304)$.

We analyzed the BTF3 expression trend, as shown in Fig. 2, the expression of BTF3 increased from distant or adjacent normal mucosa to primary CRCs stage I and II cancers $(p<0.0001)$, then decreased to stage III and IV (64\% vs. 35\%, $p=0.0004)$, or lymph node metastases (64\% vs. $29 \%, p=0.004)$. There was no significant difference between primary CRCs stage III and IV, and lymph node metastases (35 \% vs. 29\%, $p=0.522$ ).

Only a small number of cases showed nuclear staining. We regarded the cases 
with less than $5 \%$ of nuclear stained cells as negative group, and the other as positive group. Of the 42 distant normal mucosa samples, 41 (98\%) were negative, and 1 (2\%) positive. None of the 96 adjacent normal mucosa samples was positive. In the 156 primary CRCs, 150 (96\%) had negative staining, and 6 (4\%) positive staining. Regarding the 35 lymph node metastases, 33 (94\%) were negative, and 2 (6\%) positive. There was no significant difference between either of the two groups $(p>0.05)$.

BTF3 expression in relation to clinicopathological features

Cytoplasmic BTF3 expression was related to tumor site, stage and differentiation. Tumors from ascending and transverse colon were graded as proximal while tumors from descending colon and rectum were considered as distal. 39\% proximal cancers showed strong BTF3 staining, while 57\% distal cancers were strong expression, and the difference was significant ( $p=0.041$, Table 2). Regarding the tumor stage, 57\% $(12 / 21)$ was strong staining in stage I, 66\% (43/65) in stage II, 30\% (12/41) in stage III and 37\% (10/27) in stage IV. We combined stage I with II, as well as stage III with IV, and BTF3 showed stronger staining in stage I and II than that in stage III and IV (64\% vs. 35\%, $p=0.0004$, Table 2). Better differentiation cancers had a higher expression than worse differentiation ones ( $56 \%$ vs. $37 \%, p=0.031$, Table 2 ). BTF3 expression was not related to gender, age, growth pattern ( $p>0.05$, Table 2$)$, neither to patient survival ( $p=0.285$, Fig. 3). 
BTF3 and tumor-associated genes

We used Spearman correlation test to analyze the relationship of BTF3 with other molecular markers taken from previous studies performed at our laboratory. BTF3 had

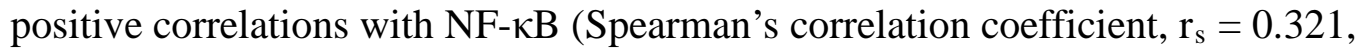
$p=0.0006), \operatorname{RAD} 50\left(\mathrm{r}_{\mathrm{s}}=0.285, p=0.0007\right), \operatorname{MRE} 11\left(\mathrm{r}_{\mathrm{s}}=0.306, p=0.0004\right) \operatorname{NBS} 1\left(\mathrm{r}_{\mathrm{s}}\right.$ $=0.210, p=0.016)$ and AEG-1 $\left(\mathrm{r}_{\mathrm{s}}=0.340, p=0.0003\right)($ Table 3$)$. 


\section{Discussion}

Transcription factors are frequently deregulated in CRC, leading to different pathophysiological effects in this malignancy. In the present study, we find that BTF3 is overexpressed in CRCs compared with distant or adjacent mucosa, and related with tumor site, stage and differentiation; it has positive correlations with tumor-associated genes NF- $\kappa$ B, RAD50, MRE11, NBS1 and AEG-1, and might influence complex signal pathways.

The cytoplasmic staining of BTF3 showed an interesting expression pattern from normal mucosa to primary CRCs stage I and II, stage III and IV, and lymph node metastases (Fig. 2). It increased from distant or adjacent normal mucosa to primary cancers and lymph node metastases. In stage I and II cancers, BTF3 reached to peak level, then decreased to stage III and IV cancers, and lymph node metastases. The expression trend supported that BTF3 overexpression was an early event in CRC development, and it seemed that BTF3 played less role in the progression of CRC.

Two distinct pathways of tumorigenesis exist in sporadic CRC, the chromosomal instability (CIN) pathway and microsatellite instability (MSI) pathway [10]. The progress of CIN involves both oncogenes and tumor-suppressor genes including chromosomes $5 q, 17 p$, and $18 q[11,12]$. It is reported that the frequency of allelic loss on the three chromosomes is more than double in distal tumors compared with proximal tumors [11]. However, as for the proximal colon, the MSI pathway is predominant, and this is reflected in the high incidence of MSI phenotype in the 
proximal colon $[13,14]$. In distal CRCs, BTF3 had a higher expression that that in proximal ones, suggesting more active actions of BTF3 in distal CRCs, and giving further support that CRCs from different anatomic site should not be assumed to be constant in their biological behavior or relative risk factors [15].

Immunohistochemistry results showed that BTF3 expressed in the both cytoplasm and nucleus, mainly in the cytoplasm, of epithelial and cancer cells in CRC. As for the protein localization, it was in line with previous report that BTF3 staining in the cytoplasm and nucleus of cancer cells, as well as in tubular complexes in PDAC [8]. The cytoplasmic staining of BTF3 was obviously up-regulated in CRC than that in distant or adjacent mucosa; unexpectedly, only a small number of cases showed nuclear staining, no significant difference between cancer tissues and distant or adjacent mucosa was found. Since the exact mechanisms of translocation from the cytoplasm to the nucleus, as well as BTF3 functions had yet to be well defined, possible explanations for this phenomenon were 1) translocation from the cytoplasm to the nucleus was aberrant during the CRC development, and caused the BTF3 storage in the cytoplasm, 2) once nuclear BTF3 exert its functions, it would be rapidly degraded, so it was difficult to detect its overexpression in nucleus, and 3) the mechanisms acting through protein activity in the cytoplasm were involved in the carcinogenesis of CRC, for example, BTF3 could bind to newly synthesized polypeptide chains that emerged from the ribosome [16]. Further studies are needed to elucidate whether only cytoplasmic overexpression of BTF3 in cancer cells is CRC specific or a common phenomenon even in other cancers. 
The functions and underlying molecular mechanisms of BTF3 are far from clear. Our primary analysis finds that the BTF3 has significant relationship with NF- $\kappa B$, RAD50, MRE11, NBS1 and AEG-1, and indicates that BTF3 might influence complex signal pathways, and affect cancer cells in CRC patients. But the correlation coefficients presented here are low, further studies are needed to experimentally verify these possible interaction signal pathways.

In conclusion, BTF3 overexpression may be an early event in CRC development, and could be useful biomarker for the early stage of CRC. BTF3 has positive correlations with NF- $\kappa$ B, RAD50, MRE11, NBS1 and AEG-1, and might influence complex signal pathways. 
Wang CJ et al.

\section{Acknowledgements}

This study was supported by grants from Swedish Cancer Foundation, Swedish

Research Council and the Health Research Council in the South-East of Sweden.

\section{Conflict of interest}

The authors declare that they have no conflict of interest 


\section{References}

1. Zheng XM, Moncollin V, Egly JM, Chambon P. A general transcription factor forms a stable complex with RNA polymerase B (II). Cell.1987; 50(3): 361-8.

2. Zheng XM, Black D, Chambon P, Egly JM. Sequencing and expression of complementary DNA for the general transcription factor BTF3. Nature. 1990; 344(6266): 556-9.

3. Deng JM, Behringer RR. An insertional mutation in the BTF3 transcription factor gene leads to an early postimplantation lethality in mice. Transgenic Res. 1995; 4(4): 264-9.

4. Li R, Liu XL, Du QF, Zhang S, Luo RC, Zhou SY. Proteome analysis of apoptotic K562 cells induced by harringtonine. Zhonghua Xue Ye Xue Za Zhi. 2004; 25(6): $323-7$.

5. Brockstedt E, Otto A, Rickers A, Bommert K, Wittmann-Liebold B. Preparative high-resolution two-dimensional electrophoresis enables the identification of RNA polymerase B transcription factor 3 as an apoptosis-associated protein in the human BL60-2 Burkitt lymphoma cell line. J Protein Chem. 1999; 18(2): 225-31.

6. Odreman F, Vindigni M, Gonzales ML, Niccolini B, Candiano G, Zanotti B, et al. Proteomic studies on low- and high-grade human brain astrocytomas. J Proteome Res. 2005; 4(3): 698-708.

7. Wang S, Lu LC, Fang WY, Yao KT. Differentially expressed transcription factor-related genes in nasopharyngeal carcinoma and nasopharyngeal tissues, Nan Fang Yi Ke Da Xue Xue Bao. 2007; 27(2): 146-9. 
8. Kusumawidjaja G, Kayed H, Giese N, Bauer A, Erkan M, Giese T, et al. Basic transcription factor 3 (BTF3) regulates transcription of tumor-associated genes in pancreatic cancer cells. Cancer Biol Ther. 2007; 6(3): 367-76.

9. Roy L, Laboissière S, Abdou E, Thibault G, Hamel N, Taheri M, et al. Proteomic analysis of the transitional endoplasmic reticulum in hepatocellular carcinoma: an organelle perspective on cancer. Biochim Biophys Acta. 2010; 1804(9): 1869-81.

10. Dunican DS, McWilliam P, Tighe O, Parle-McDermott A, Croke DT. Gene expression differences between the microsatellite instability (MIN) and chromosomal instability (CIN) phenotypes in colorectal cancer revealed by high-density cDNA array hybridization. Oncogene. 2002; 21(20): 3253-7.

11. Delattre O, Olschwang S, Law DJ, Melot T, Remvikos Y, Salmon RJ, et al. Multiple genetic alterations in distal and proximal colorectal cancer. Lancet. 1989; 2(8659): 353-6.

12. Gervaz P, Bouzourene H, Cerottini JP, Chaubert P, Benhattar J, Secic M, et al. Dukes B colorectal cancer: distinct genetic categories and clinical outcome based on proximal or distal tumor location. Dis Colon Rectum. 2001; 44(3): 364-72.

13. Miyakura Y, Sugano K, Konishi F, Ichikawa A, Maekawa M, Shitoh K, et al. Extensive methylation of hMLH1 promoter region predominates in proximal colon cancer with microsatellite instability. Gastroenterology. 2001; 121(6): 1300-9.

14. Thibodeau SN, Bren G, Schaid D. Microsatellite instability in cancer of the proximal colon. Science; 1993; 260(5109): 816-9. 
15. Li FY, Lai MD. Colorectal cancer, one entity or three. J Zhejiang Univ Sci B. 2009; 10(3): 219-29.

16. Rospert S, Dubaquié Y, Gautschi M. Nascent-polypeptide-associated complex. Cell Mol Life Sci. 2002; 59(10): 1632-9. 
Wang CJ et al.

\section{Figure legends}

A

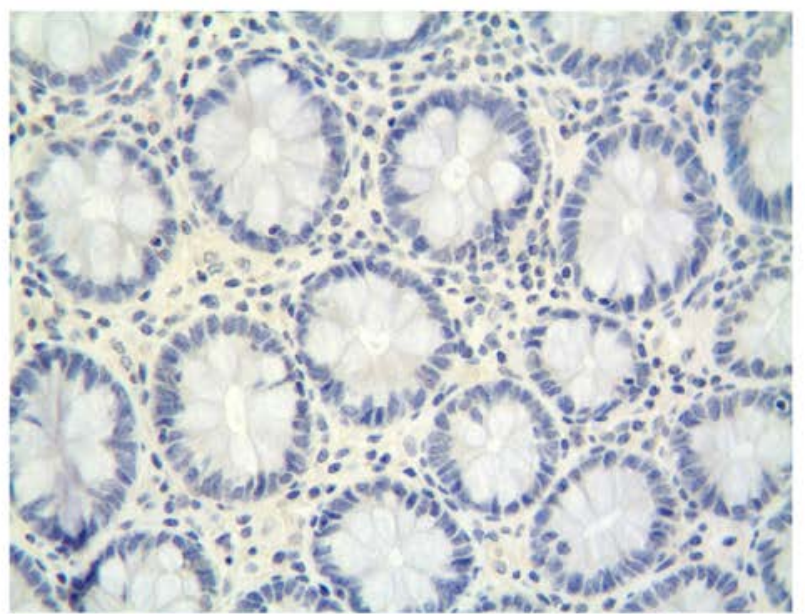

B

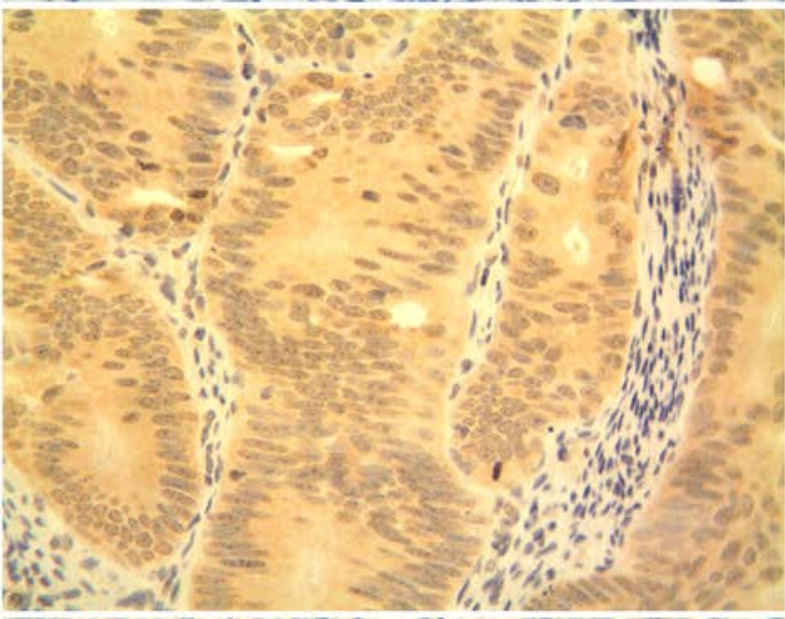

C

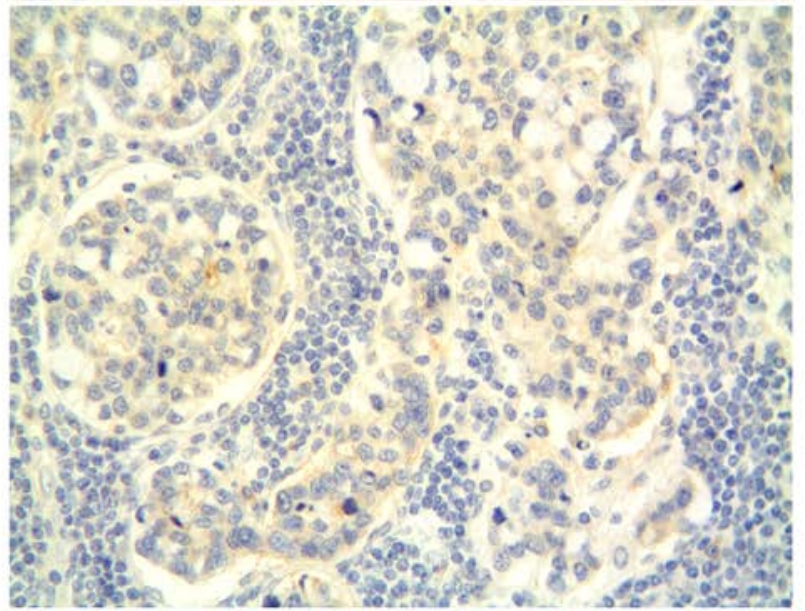

Figure 1. Expression of BTF3 in distant normal mucosa (A, negative), primary

colorectal cancers (B, strong) and lymph node metastases (C, weak) 


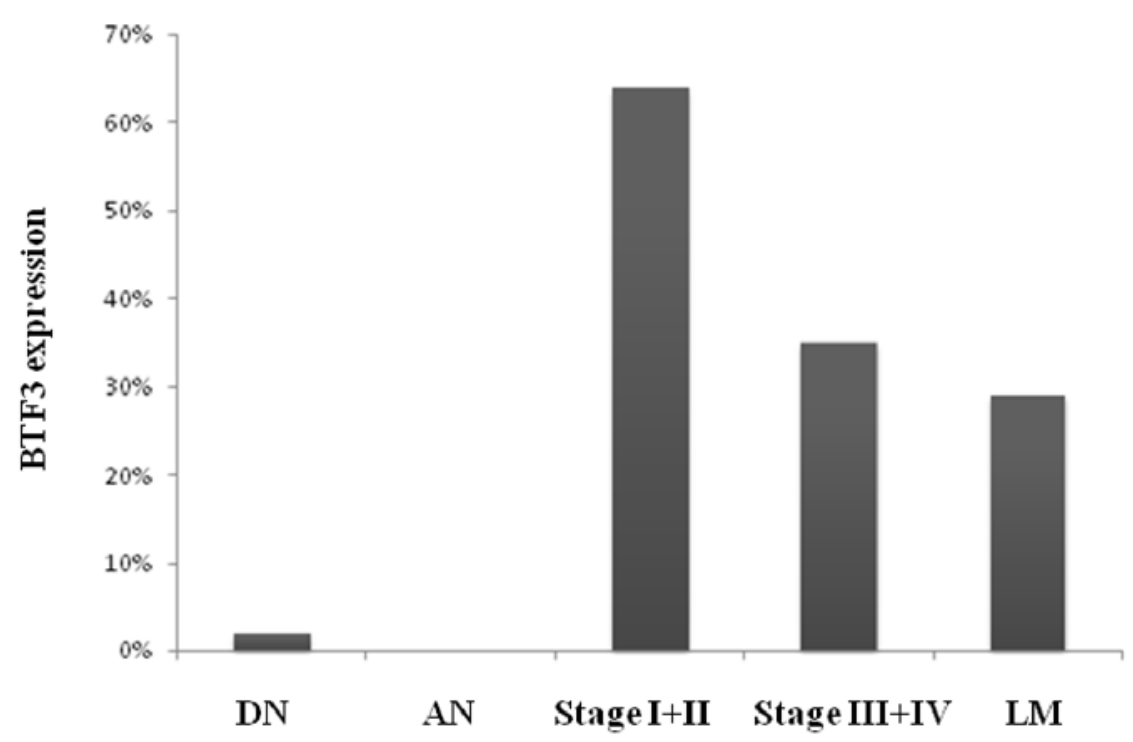

Figure 2. BTF3 expression trend in normal mucosa, primary colorectal cancers and lymph node metastases (DN, distant normal mucosa; AN, adjacent normal mucosa; LM, lymph node metastases)

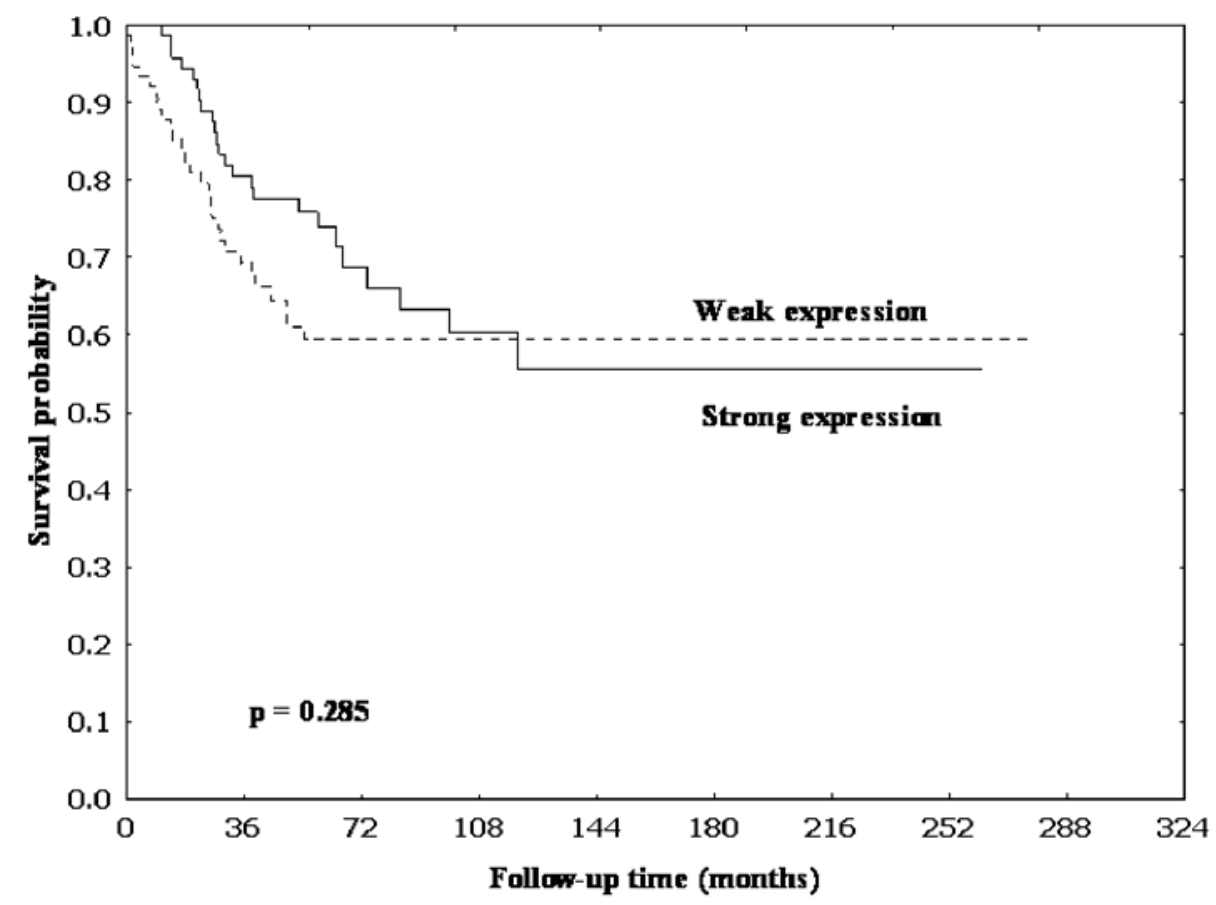

Figure 3. BTF3 expression in relation to survival in colorectal cancer patients 
Table 1 BTF3 expression in distant normal mucosa, adjacent normal mucosa, primary colorectal cancers and lymph node metastases

\begin{tabular}{lccc}
\hline Category & Weak & Strong & $p$-value* \\
\hline Distant normal mucosa & $41(98 \%)$ & $1(2 \%)$ & $<0.0001^{\dagger}$ \\
Adjacent normal mucosa & $96(100 \%)$ & $0(0)$ & $<0.0001^{\dagger}$ \\
Primary colorectal cancers & $77(49 \%)$ & $79(51 \%)$ & \\
Lymph node metastases & $25(71 \%)$ & $10(29 \%)$ & $0.018^{\ddagger}$ \\
\hline
\end{tabular}

*Compared with primary colorectal cancers; † Fisher’s exact test; ${ }^{\ddagger} \chi^{2}$ test 
Table 2 BTF3 expression in relation to clinicopathological features

\begin{tabular}{|c|c|c|c|}
\hline \multirow[b]{2}{*}{ Varibale } & \multicolumn{2}{|c|}{ Cytoplasmic staining } & \multirow[b]{2}{*}{$p$-value } \\
\hline & Weak & Strong & \\
\hline Gender & & & 0.863 \\
\hline Male & $42(50 \%)$ & $42(50 \%)$ & \\
\hline Female & 35 (49\%) & 37 (51\%) & \\
\hline Age (year) & & & 0.725 \\
\hline$<71$ & 31 (48\%) & 34 (52\%) & \\
\hline$\geq 71$ & $46(51 \%)$ & 45 (49\%) & \\
\hline Tumor site & & & 0.041 \\
\hline Proximal & 27 (61\%) & 17 (39\%) & \\
\hline Distal & 47 (43\%) & 62 (57\%) & \\
\hline Stage & & & 0.0004 \\
\hline $\mathrm{I}+\mathrm{II}$ & $31(36 \%)$ & $55(64 \%)$ & \\
\hline III+IV & 43 (65\%) & 23 (35\%) & \\
\hline Growth pattern & & & 0.390 \\
\hline Expansive & $41(48 \%)$ & 45 (52\%) & \\
\hline Infiltrative & 34 (55\%) & $28(45 \%)$ & \\
\hline Differentiation & & & 0.031 \\
\hline Better & $48(44 \%)$ & $61(56 \%)$ & \\
\hline Worse & $29(63 \%)$ & 17 (37\%) & \\
\hline
\end{tabular}


Table 3 BTF3 correlates with some tumor- associated genes in colorectal cancer

\begin{tabular}{lccc}
\hline Gene name & $\mathrm{N}$ & $\mathrm{r}_{\mathrm{s}}{ }^{*}$ & $p$-value \\
\hline NF-kB & 111 & 0.321 & 0.0006 \\
RAD50 & 138 & 0.285 & 0.0007 \\
MRE11 & 130 & 0.306 & 0.0004 \\
NBS1 & 130 & 0.210 & 0.016 \\
AEG-1 & 111 & 0.340 & 0.0003 \\
\hline
\end{tabular}

*Spearman's correlation coefficient 